\title{
Return to Nature: How media art heals?
}

\author{
Tony Langford \\ Fovolab \\ Cardiff Metropolitan University, Wales \\ tolangford@cardiffmet.ac.uk
}

\begin{abstract}
This paper contextualises developments in media art and electronic visualisation with the 'Consciousness Hacking' movement, and the potential for technology to improve psychological, emotional and spiritual wellbeing. The narrow focus of attention demanded by many contemporary media devices, such as smart phones and some immersive technologies, may be harmful to cognitive functioning and emotion regulation. These technologies are arguably a distraction that diminishes our natural capacity for rich sensory experience. Conversely, artists and researchers have experimented with new media in an attempt to challenge and engage our sensory experience, in order to re-connect us to nature. Trials comparing simulated natural environments with actual nature are reviewed within the context of their potential to restore attention. Do multi-sensory media artworks and visualisations re-connect us with the natural world, or remove us further from it? The potential benefits of technology are assessed as a tool for ecotherapy in mediated environments and for new models of mental healthcare and wellbeing. Comparison is made between 'Cyberdelics' virtual applications, which may narrow or distract the focus of attention, and works using more natural systems, which engage with the periphery and interconnectedness of the senses. The interventions are assessed in their efficacy to affect change in psychological and physiological states, and as a non-pharmacological enhancement with therapeutic applications. Transformation and healing is possible when media art and technology are created and disseminated with mindful intention.
\end{abstract}

Media. Art. Technology. Consciousness. Attention. Wellbeing. Nature. Visualisation. Cyberdelics. Mindfulness

\section{INTRODUCTION}

The effects of technology, particularly social media, on human health and wellbeing are currently being rigorously debated at both an academic and political level. The UK Parliament has recently launched an enquiry into the growth of immersive and addictive technologies, and gaming addiction can now be treated on the NHS after the World Health Organisation classified it as a medical disorder. The narrow focus of attention demanded by many contemporary media devices, such as smart phones and some immersive technologies, may be harmful to cognitive functioning, emotion regulation and visual perception, (Lim 2017; Wilmer, Sherman \& Chein,2017), as well as musculoskeletal and metabolic systems (Laatar 2017).

Conversely a new generation of artists and researchers have been experimenting with the ever more accessible and expanding possibilities of new technologies in an attempt to challenge and engage human sensory experience, in order to reconnect us to each other and to nature. In recent years the 'Consciousness Hacking' movement has emerged as a global community focused on exploring, designing, and using technologies which facilitate individual and collective awakening, in the belief that modern technology driven by science has the potential to support psychological, emotional, and spiritual wellbeing (Siegel \& Gwyn 2016).

There is already a growing body of evidence to support the effects of arts and cultural engagement on health and wellbeing, and how the arts can improve healthcare environments. An All-Party Parliamentary Group on Arts, Health and Wellbeing (APPGAHW) has been formed with the aim to improve awareness of the benefits that the arts can bring to health and wellbeing, and the Inquiry Report, Creative Health: The Arts for Health and Wellbeing has examined the benefits of participatory arts programmes, arts therapy, and arts on prescription. A common theme they all share is that they involve the active engagement of individuals and communities. 
Meanwhile there is also significant evidence that exposure to natural environments is associated with mental health benefits. Proximity to greenspace has been associated with lower levels of stress (Thompson et al. 2012), reduced symptomology for depression and anxiety (Beyer et al. 2014), sustained improvements in mental health (Alcock et al. 2014), and the promotion of children's learning (Kuo, Barnes \& Jordan 2019).

The focus and scope of this paper is to explore the evidence for how and whether developments in media art and electronic visualisation support mental health and wellbeing, and in particular the extent to which art and technology can re-connect the link between mind, body and the natural world.

The link between mind and nature can be traced back to spiritual traditions such as Buddhism and the idea that our responses to the environment are connected to how we have evolved as a species. Nature provides a live and dynamic environment, which leads to revelatory sensory experiences. Based on preference ratings for different landscapes, people tend to prefer natural environments more than built environments, and they prefer built environments with natural features such as water and trees, more than built environments without biophilic elements (Kaplan \& Kaplan 1989).

Biophillia was a term originally coined by the naturalist Edward O. Wilson (1984), which he defined as the innate tendency to focus on 'life and lifelike processes'. Natural environments benefit from what Rachel and Stephen Kaplan termed soft fascination, which refers to scene content that automatically captures attention while simultaneously eliciting feelings of pleasure. But do people need to physically interact with nature, or can they receive the apparent health benefits through passive exposure?

\section{REAL VS VIRTUAL NATURE}

Even a minimal connection with nature, such as looking at it through a window, can be beneficial. Peter Kahn (2011) developed a research programme to explore whether interacting with lifelike nature - or 'technological nature' - could provide the same psychological benefits as actual nature. He showed how the heart rates of participants exposed to the sight of real nature decreased more quickly than those of subjects looking at an electronic visualisation of nature on a monitor screen.

In another comparison of the restorative effect of a natural environment with that of a simulated natural environment (Kjellgren \& Buhrkall 2010), participants described the natural environment in terms of intensified sensory perception, feelings of harmony and union with the surroundings, and 'here-and-now' thinking. These are some of the hallmarks of an altered state of consciousness (ASC), a state of reality that is separate from an individual's normal day-to-day reality and may be characterised by alterations in cognition and perception, feelings of rejuvenation, and a shift away from logical thinking and directed attention, to more intuitive, creative thinking.

Meanwhile the simulated natural environment allowed people to 'relax and feel good, but fails to stimulate sensory perceptions, does not induce a feeling of connection with nature, and does not promote a sense of energy and awakening'.

However, attention restoration effects have been observed after participants simply watch films or images that depict natural scene content (Berto 2005), implying that direct physical engagement with nature may be unnecessary to promote positive restoration effects. Sue Thomas (2013) created the term 'technobiophilia' to describe 'the innate attraction to life and lifelike processes as they appear in technology'.

To what extent are virtual environments able to replicate the complexity of nature for the purposes of attention restoration? An investigation into the influence of immersion revealed that a larger screen size on which the participants viewed the content resulted in stronger restorative potential of a nature scene (de Kort et al. 2006). Stress recovery has been shown to be facilitated by the addition of sounds of nature (birdsong and a babbling brook) to a virtual forest in a CAVE system with three rear-projected walls and a floor projection (Annerstedt et al. 2013). Is a simulated natural environment more restorative when viewers are more immersed in it? In recent years several artists, researchers and creatives have explored this question by developing multisensory mediated environments.

\section{MEDIA ART AND NATURE}

Over many years as a co-founder and director of Kinetica Museum, I have experienced first-hand how multi-disciplinary art can capture and engage an audience's attention. Digital Forest is a collaborative project by media artist Madi Boyd and cognitive scientist Polly Dalton, which aims to explore the psychological benefits of immersive art, and whether it can re-create the benefits of 'soft fascination' provided by natural environments. The project was shown in summer 2018 at Royal Holloway, where visitors experienced an installation using sculpture and sound in addition to moving image, with the aim of conveying the multi-sensory 
complexity of a forest. The audience encountered projected geometric patterns, dispersed sounds and tactile encounters. After the experience they performed a task based on 'Attention Restoration Theory' (Kaplan; 1995), which demonstrates that walking through a forested or green space reduces stress and restores attentional resources.

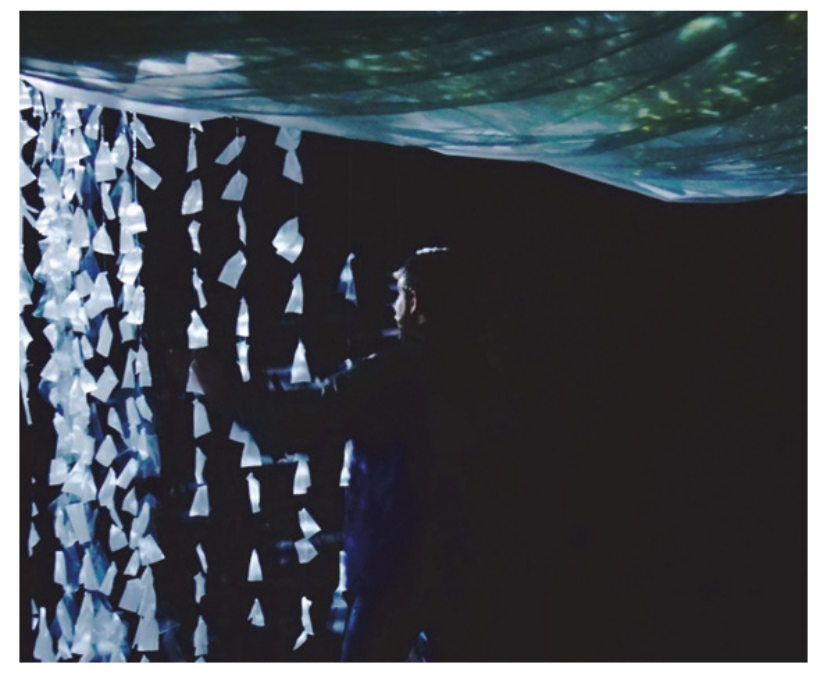

Figure 1: Digital Forest.

Qualitative feedback gathered from participants indicate that the experience was peaceful, relaxing and rejuvenating, however the data from the task did not show the hypothesised levels of restoration and concentration. The project is undergoing a reevaluation of its methodology, and has highlighted the difficulties of reliably measuring and recording the impact of multi-sensory works.

Marshmallow Laser Feast are another group of artists exploring how and whether we can reconnect to nature using technology. Their work 'Laser Forest' consists of 120 metal rods, each attached to a laser, that produce light and sound inspired from the natural world. In recent years they have experimented with the medium of virtual reality combined with other multi-sensory technologies in order to immerse viewers into perspectives of the natural and animal world. 'In The Eyes of the Animal', when it originally launched in 2015 , was set in a real forest so that viewers could directly experience the landscape through the eyes of woodland creatures.

Their 2019 installation 'We Live in an Ocean of Air' brings the forest back indoors, and uses a tracking system that allows participants to confidently explore the environment including through a giant sequoia tree. Breath and heart sensors track realtime breathing, while scent dispersal systems and subtle wind machines add further dimensions to the true multi-sensory experience. A clear aim of the MLF installation is to encourage viewers to reflect on their dependence and responsibility to the organisms with which we share the planet, in such a way that inspires wonder towards the natural world.

Initial qualitative feedback from visitors to these installations has indicated that they have a high initial impact on visitors and generate feelings of awe and wonder towards nature (Segreto 2019). Further data has been collected and is awaited on how people are emotionally affected by the experience, and whether there are long-term restorative benefits.

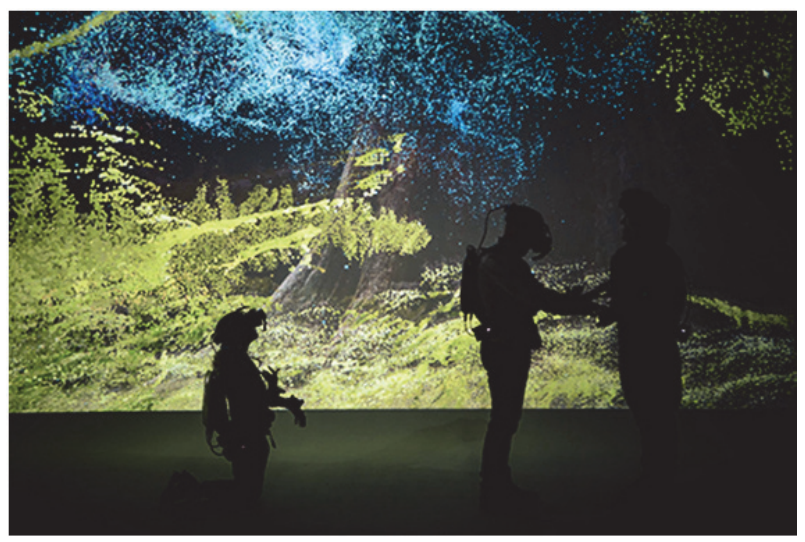

Figure 2: We Live in an Ocean of Air.

Does the trend towards replicating nature using increasingly sophisticated technologies make people more likely to settle for experiencing nature second-hand, as opposed to venturing out into the real thing? As we move further into a technological future, do we move further away from the elemental forces that shaped our minds, and if so how do we re-connect with them?

\section{TECHNOLOGY AND ECOTHERAPY}

Interdependence of the mind and nature is at the foundation of ecotherapy, a union between the ideas behind ecopsychology and psychotherapy, and a form of treatment that aims to improve mental and physical wellbeing through engaging with nature.

In what ways can art and technology enhance or extend the benefits of ecotherapy? Numerous virtual reality applications have been developed using the concept of how immersion in nature can be beneficial for wellbeing. The Oculus has an application "Guided Meditation VR" with 30 different environments such as forests, mountains and waterside scenes. The emphasis is firmly on how the technology transports the user to another world (Loke Hale 2018).

Several studies have shown how virtual reality is an effective tool for relaxation and distraction in the treatment of pain relief, and as exposure therapy 
for the treatment of anxiety disorders and phobias (Valmaggia et al. 2016). A recent review of the use of nature in virtual reality applications for therapy concluded that while real-world nature is preferred, VR use could be an alternative in cases when in vivo contact with nature is not possible (White et al. 2018).

A company in the Netherlands has developed a virtual application 'Swimming with Dolphins' as a drug-free medicine that is showing potential in the treatment of both psychological and physical conditions. The treatment is however most effective when combined with hydrotherapy, in other words swimming in real water while experiencing the dolphins in VR (Sjollema \& Brada 2017).

Another Dutch company, Sensiks, has also explored the therapeutic possibilities of a virtual reality experience that stimulates multiple senses and bodily sensations. They have developed a 'Sensory Reality Pod', where wind, smell and temperature are combined with audiovisual content (Holger 2018). Physiological metrics of the user can be measured and the sensory stimulation adjusted accordingly. Their Pods have been applied for relaxation as well as in mental health and for people with disabilities. Participants in the later stages of dementia have also shown positive changes in mood and behaviour, and also an increase in attention to their surroundings.

While Sensiks' focus is on stepping into another (sensory) reality, the Be-box is another immersive space but its emphasis is more on developing the user's internal state, as opposed to stimulating multiple external senses. Its focus is 'where art, science and technology meet mind, body and soul' and is designed as an environment for meditation that guides users into moments of presence. Bebox have collaborated with the artist Based Upon to create sculptural and audiovisual representations of nature that harmonise the energetic patterns of the mind.

Wavepaths, a multidisciplinary team of scientists, technologists, designers, psychotherapists and artists, have opened a similar immersive space in London in 2019. Their aim is to develop 'Experience as Medicine', provided through both mobile tools and applications as well as urban spaces combining multisensory media, architecture, design and psycho-emotional techniques. Wavepaths was developed by Mendel Kaelen, who has researched the therapeutic use of sound and music within psychedelic-assisted psychotherapy (Barrett, Preller \& Kaelen 2018), and is now developing a new category of psychotherapeutic tools for healthcare. He envisions a radical new model of mental healthcare and wellbeing, and is developing research projects to assess the neurobiological and psychological mechanisms of how and whether generative music and light art can facilitate transformative experiences.

\section{SOUND, LIGHT AND VIBRATION}

The ways in which sound affects us have been literally brought to light through the study of cymatics, and the connection between frequencies in our bodies and in the natural world. The recurring and vibrational geometry represented by cymatics images give validity to the rejuvenating power of spending time in nature. Everything is connected once we realise the essence of frequency vibration.

Hans Jenny first started experimenting with vibrating frequencies and their effect on water and solids and published a book showing photos of the results (Jenny 2001). Water, which makes up $70 \%$ of the human body, took on extraordinary geometric forms in the experiments.

The therapeutic value of cymatics is still a relatively uncharted field of scientific inquiry, but the basic principle of using sound for healing is that every part of the body is in a state of vibration. Artists such as Evgenia Emets and Rachael Linton have experimented with the possibilities of cymatics, believing that it holds an important function in the development of holistic healing. Research is also emerging into the effects of sound and light vibration from live performance art (Almena 2018).

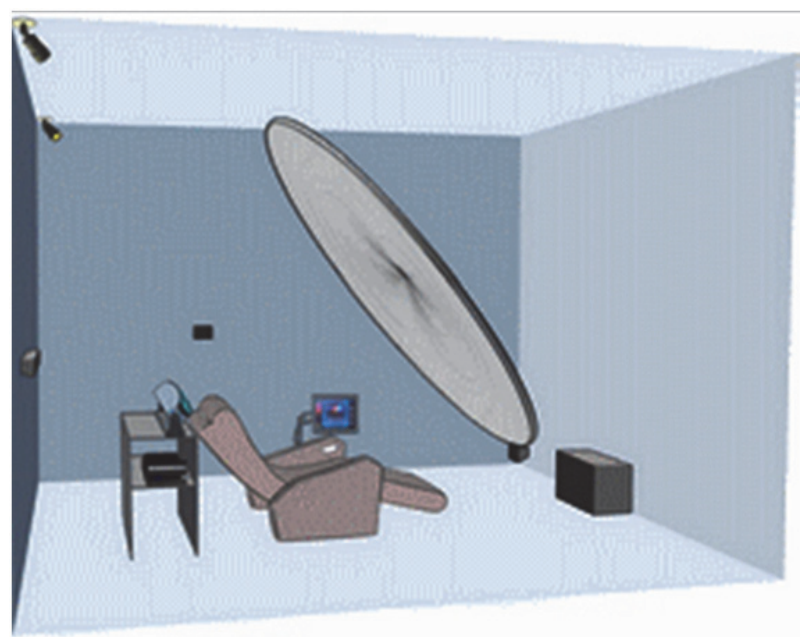

Figure 3: The Sensora.

An installation which has been used in therapy and combines sound, light and kinaesthetic vibration is Sensortech's 'Sensora'. The frequencies and pulses of light interact with the physiological rhythms of brainwaves, heartbeat and breathing. Clinical evidence has demonstrated how the light has a positive effect in developing well-being and 
relaxation, as well as increased focus (Ross, Guthrie \& Dumont 2013).

Flickering light has been used to stimulate perception since the days of Nostradamus in 1564 (Haill 2013). Using EEG monitoring the artist Lucianna Haill has investigated the synchronisation between frequencies of brainwaves and 'Dreammachines', kinetic lights invented by Brion Gysin with a perforated, spinning template that can induce alpha waves and altered states of consciousness in the viewer.

The healing potential of pulsing light prompted a multi-disciplinary team at Light Attendance in Austria to develop Lucia no.3, a meditation lamp that navigates 9 light sources to optically stimulate the brain. A study from 2015 showed that the lamp was an effective tool in inducing a temporary dissociative state (Schwartzman 2015). The light is experienced with the eyes closed, and participants often report seeing imagery or geometry that is borrowed from nature.

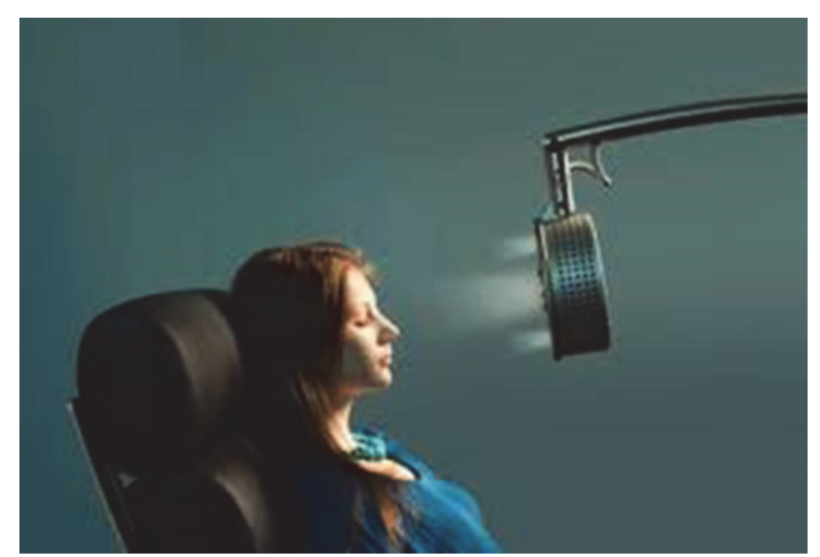

Figure 4: Lucia no.3.

\section{TECHNOLOGY, PRESENCE AND HEALING}

There is increasing evidence of the potential for these devices and installations to alter consciousness and unlock a more creative, intuitive mind state in the participants who experience them. What is less clear is how best to apply these technologies within the therapeutic context. Although they do offer an alternative to both pharmacological and psychoactive substances, 'cyberdelics' are not directly comparable to psychedelics, since there is currently no evidence to equate the effect of technology on consciousness in the transformative way that has been proven with the use of LSD (Carhart-Harris et al. 2016) or plant medicine such as ayahuasca (Palhano-Fontes et al. 2019).

While on-going trials are likely to result in psychedelics becoming more widely accepted and used in therapy, it is important that further evidence is compiled in support of technological risk-free alternatives and their impact on attention, whether in the form of restoration or distraction. Questions remain as to whether many of these technologies can bring about the genuine presence, connection and self-awareness that leads to transformational experience, instead offering external distraction or escape that may bring relaxation or temporary relief, but not necessarily long-lasting healing from past trauma, pain or suffering.

Commonly technologies are 'unnatural', in that they do not adequately represent what we perceive naturally through our senses. Companies and brands such as Otherworld (opening in London in 2019) now seek to immerse consumers in the experience of a natural environment, which is in reality an artificial one. Intuition, imagination and instinct may be left behind in such a hypermediated world.

The alternative is more natural media (Smith et al. 2016) and technologies that are modelled on human perception, cognition, awareness and proprioception, and take into account how our bodies interact with the environment, and how different sensory modalities interact with each other. FOVO (Field of View Opened) is a form of natural media that provides a more naturalistic and immersive form of imaging (Pepperell et al. 2019) than is provided by conventional cameras and computer graphics systems, which universally rely on linear perspective, invented by artists during the Renaissance but long seen as ineffective for representing the full scope of human vision (Pepperell and Haertel 2014).

In his 2019 book 'Team Human', Douglas Rushkoff comments how while in pre-civilised times people used to believe in circles, they later came to believe in lines and hence became disconnected from the cycles and renewal of day, night, moon and season: 'spirituality has become less of a state of being than yet another goal to attain in the future' (Rushkoff 2019).

The image geometry of Fovo is reflective of the curvature found in nature, in contrast to the straight lines of urban environments. It incorporates peripheral sensory information to create a more naturalistic perspective, and has shown measurable benefits in the broadening of attention, and as a tool for mindfulness training (Langford et al. 2018).

Sense of presence and immersion are often seen as interchangeable, however there is a clear distinction between when the user is present with their own experience, their thoughts as well as body sensations, and when they are immersed (or, 
as can be argued, distracted) in another world or reality. The former is more likely to lead to transformational experiences and healing than the latter, by creating a safe context of self-compassion and social connection whereby the parasympathetic nervous system is activated (Porges 2009)

Creators of immersive experiences are beginning to consider this 'Mindful Design' approach, where the welfare and wellbeing of the user is given equal consideration to the design of the experience itself, using a neurocognitive model of mindful attention (Hasenskamp et al. 2012; Russell 2017). This approach is likely to lead to intentions that are intuitive, creative and empathetic, as opposed to the logical, analytical, accountable approach more commonly associated with technology creation and distribution (McGilchrist 2012).

Early in 2019 the Cyberdelic Society organised an event to set intention around how immersive technologies (Extended Reality) can be used to measure the impact of humanity on nature and the environment (Extinction Rebellion). Their aim is to maximise the collective knowledge and energy from the event in order to co-create an optimal solution to the environmental crisis using XR technologies. Such a call to action is very much in spirit with Rushkoff's 'Team Human' manifesto to regenerate the social bonds for human connection to make a positive impact on the planet.

\section{CONCLUSION}

Technology by itself is unlikely to provide the answers, particularly in an era of information overload where it tends to externalise or distract our attention, and divide rather than unite us. But harnessed with the right intention and creativity, technology does have the power to connect and transform. And so it is with media art: energetic vibration radiates from artworks that have been created with the intention to connect us to nature. They have the potential to awaken the life force that exists within us.

The extent to which they can directly alter or transform consciousness is less clear. Media and technology may never provide a substitute for more traditional, relational therapy, but it can be a gateway to further healing and understanding. The benefits of real nature will always outweigh any technological representation, but ultimately media art can be a 'lifelike process' that shows us the way towards the complexity, richness and variety of life that nature itself provides.

Art and technology can have a demonstrable impact on mental health and wellbeing by shifting perspectives and changing engrained systems and patterns of thinking. Herein lies the connection to the inside-out perspective of the Consciousness Hacking movement, which aims to change our relationship to the world, rather than the world itself. This is a return to a way of being where there is no barrier or separation from the nature within and around us, and a recognition of what spiritual traditions have been advocating for thousands of years - that true healing lies less in our external circumstances, and more in our relationship to ourselves.

\section{REFERENCES}

Alcock, I., White, M. P., Wheeler, B. W., Fleming, L. E. and Depledge, M. H. (2014) Longitudinal effects on mental health of moving to greener and less green urban areas. Environ. Sci. Technol. 48, 1247-1255.

Almena, M. (2018) Transcendence: Can live performance art in combination with interactive technology induce altered states of consciousness? In: Proceedings of the Conference on Electronic Visualisation and the Arts (195-202), BCS London.

Annerstedt, M., Jönsson, P., Wallergård, M., Johansson, G., Karlson, B., Grahn, P., Hansen, A.M. and Währborg, P. (2013) Inducing physiological stress recovery with sounds of nature in a virtual reality forest, Physiology \& Behavior, 118 pp.240-250

Barrett, F. S., Preller, K., and Kaelen, M. (2018) Psychedelics and music: neuroscience and therapeutic implications, International Review of Psychiatry, 30(8):1-13

Berto, R. (2005) Exposure to restorative environments helps restore attentional capacity, Journal of Environmental Psychology, 25(3), pp.249-259

Beyer, K. M. M., Kaltenbach, A., Szabo, A., Bogar, S., Nieto, F. J. and Malecki, K. M. (2014) Exposure to neighbourhood green space and mental health: Evidence from the survey of the health of Wisconsin. Int. J. Environ. Res. Public Health 11, 3453-3472.

Carhart-Harris, R. L. et al. (2016) Neural correlates of the LSD experience revealed by multimodal neuroimaging.

de Kort, Y. A. W., Meijnders, A. L., Sponselee, A. A. G. and IJsselsteijn, W.A., (2006), What's wrong with virtual trees? Restoring from stress in a mediated environment, Journal of Environmental Psychology, 26 pp.309-320.

Haill, L. (2013) ICT \& Art Connect: Revelations by Flicker, Dreamachines and Electroencephalographic signals in art, Goldsmiths. 
Hasenkamp, W., \& Barsalou, L., Effects of Meditation Experience on Functional Connectivity of Distributed Brain Networks. Frontiers in Human Neuroscience 6 (2012).

Holger, D. (2018), This 'Sensory Reality Pod' Will Make You Feel VR.

https://vrscout.com/news/sensory-reality-pod-feelvr, (retrieved 5 Mar 2019).

Jenny, H. (2001) Cymatics: A Study of Wave Phenomena \& Vibration. MACROmedia Publishing.

Kahn, P. (2011) Technological Nature: Adaptation and the Future of Human Life. MIT Press.

Kaplan, R. and Kaplan, S. (1989) The Experience of Nature: A Psychological Perspective. Cambridge University Press, New York.

Kaplan, S., (1995), The restorative benefits of nature: Toward an integrative framework, Journal of Environmental Psychology, 15 (3), pp.169-182.

Kinetica Museum (2019) http://www.kineticamuseum.org (retrieved 1 March 2019).

Kjellgren, A. and Buhrkall, H. (2010) A comparison of the restorative effect of a natural environment with that of a simulated natural environment, Journal of Environmental Psychology, 30(4), pp.464-472.

Kuo, M., Barnes, M. and Jordan, C. (2019) Do Experiences With Nature Promote Learning? Converging Evidence of a Cause-and-Effect Relationship. Frontiers in Psychology, 10.

Laatar, R., Kachouri, H., Borji, R., Rebai, H. and Sahli, S. (2017), The effect of cell phone use on postural balance and mobility in older compared to young adults, Physiology \& Behavior, 173, pp.293297.

Langford, T., Burleigh, A., Ruta, N. and Pepperell, R. (2018) New immersive media to broaden attention and awareness. In: Proceedings of the Conference on Electronic Visualisation and the Arts (323-330). BCS London.

Lim, J., Chang, S.H., Lee, J. and Kim, K. (2017) Effects of smartphone texting on the visual perception and dynamic walking stability. Journal of exercise rehabilitation, 13(1), p.48.

Loke Hale, J. (2018) I Meditated In Virtual Reality \& It Literally Took Me To Another Place. https://www.bustle.com/p/i-meditated-in-virtualreality-it-literally-took-me-to-another-place-9869860 (retrieved 14 Mar 2019).

McGilchrist, I. (2012) The Master and His Emissary: The Divided Brain and the Making of the Western World.
Palhano-Fontes, F., Barreto, D., Onias, H., Andrade, K., Novaes, M., Pessoa, J. and Araújo, D. (2019) Rapid antidepressant effects of the psychedelic ayahuasca in treatment-resistant depression: A randomized placebo-controlled trial. Psychological Medicine, 49(4), 655-663.

Pepperell, R., Burleigh, A., Ruta, N. and Langford, T. (2019) Fovo - a Flexible Realtime Computer Graphics Rendering Process, Electronic Visualisation in the Arts 2019.

Pepperell, R. and Haertel, M. (2014). Do artists use linear perspective to depict visual space? Perception, 43 (5), pp.395-416.

Porges, S. (2009) The polyvagal theory: New insights into adaptive reactions of the autonomic nervous system, Cleve Clin J Med. 76 (Suppl 2): S86-S90.

Ross M. J., Guthrie, P., Dumont, J. C. (2013) The impact of modulated, coloured light on the autonomic nervous system, Adv Mind Body Med, 27(4): 7-16.

Russell, T. (2017) \#What Is Mindfulness?, Watkins, London.

Rushkoff, D., (2019) Team Human. W.W. Norton \& Company, New York.

Schwartzman, D. (2015) EEG Functional Connectivity and Phenomenology of Induced Dissociative States. University of Sussex.

Segreto, G. (2019) Review - We Live in an Ocean of Air by Marshmallow Laser Feast at Saatchi Gallery.

https://www.thelondonmagazine.org/review-we-livein-an-ocean-of-air-by-marshmallow-laser-feast-atsaatchi-gallery (retrieved 5 Mar 2019).

Siegel, M. and Gwyn, G., (2016) The Frontier of Consciousness Hacking.

http://www.cohack.life/posts/happenings-at-thefrontier-of-consciousness-hacking (retrieved 14 Jan 2019)

Sjollema and Brada (2017) Wild Dolphin Virtual Reality, a drug-free medicine. https://thedolphinswimclub.com/medicalvr/casestudies-medical-vr (retrieved 14 Mar 2019).

Smith, C. H., Rasool, J., Lambert, N., Pepperell, R., Langford, T., Korkovelou, L. and Burleigh, A. (2017) Natural Media. In: Proceedings of the Conference on Electronic Visualisation and the Arts (258-263), BCS London.

Thomas, S. (2013) Technobiophilia: Nature and Cyberspace. Bloomsbury Academic, London 
Thompson, C. W., Roe, J., Aspinall, P., Mitchell, R., Clow, A., and Miller, D. (2012) More green space is linked to less stress in deprived communities: evidence from salivary cortisol patterns. Landscape Urban Plann. 105, 221-229.

Valmaggia, L. R., Latif, L., Kempton, M.J. and RusCalafell, M. (2016) Virtual Reality in the Psychological Treatment for Mental Health Problems: An Systematic Review of Recent Evidence, Psychiatry Research 236: 189-95.
White, M. P., Yeo, N. L., Vassiljev, P., Lundstedt, R., Wallergård, M., Albin, M. and Lõhmus, M. (2018) A prescription for "nature" - the potential of using virtual nature in therapeutics. Neuropsychiatric disease and treatment, 14, 3001-3013.

Wilmer, H. H., Sherman, L. E. and Chein, J. M. (2017) Smartphones and Cognition: A review of research exploring the links between mobile technology habits and cognitive functioning. Frontiers in Psychology, 8.

Wilson, E. O., (1984) Biophilia. Harvard University Press, Cambridge. 